\title{
The Electromagnetic Counterpart of the Binary Neutron Star Merger LIGO/Virgo GW170817. I. Discovery of the Optical Counterpart Using the Dark Energy Camera
}

M. Soares-Santos ${ }^{1,2}$, D. E. Holz ${ }^{3,4}$, J. Annis ${ }^{2}$, R. Chornock ${ }^{5}$, K. Herner ${ }^{2}$, E. Berger ${ }^{6}$, D. Brout ${ }^{7}$, H.-Y. Chen ${ }^{4}$, R. Kessler ${ }^{4}$, M. Sako ${ }^{7}$, S. Allam $^{2}$, D. L. Tucker ${ }^{2}$, R. E. Butler ${ }^{2,8}$, A. Palmese ${ }^{9}$, Z. Doctor ${ }^{4}$, H. T. Diehl ${ }^{2}$, J. Frieman ${ }^{2,4}$, B. Yanny ${ }^{2}$, H. Lin ${ }^{2}$, D. Scolnic ${ }^{4}$, P. Cowperthwaite ${ }^{6}$, E. Neilsen ${ }^{2}$, J. Marriner ${ }^{2}$, N. Kuropatkin ${ }^{2}$, W. G. Hartley ${ }^{9,10}$, F. Paz-Chinchón ${ }^{11}$, K. D. Alexander ${ }^{6}$,

E. Balbinot ${ }^{12}$, P. Blanchard ${ }^{6}$, D. A. Brown ${ }^{13}$, J. L. Carlin ${ }^{14}$, C. Conselice ${ }^{15}$, E. R. Cook ${ }^{14,16}$, A. Drlica-Wagner ${ }^{2}$, M. R. Drout ${ }^{17,71}$, F. Durret ${ }^{18}$, T. Eftekhari ${ }^{6}$, B. Farr ${ }^{4}$, D. A. Finley ${ }^{2}$, R. J. Foley ${ }^{19}$, W. Fong ${ }^{20,72}$, C. L. Fryer ${ }^{21}$, J. García-Bellido ${ }^{22}$, M. S . S. Gill ${ }^{23}$, R. A. Gruendl ${ }^{11,24}$, C. Hanna ${ }^{11,25}$, D. Kasen ${ }^{26}$, T. S. Li $^{2}$, P. A. A. Lopes ${ }^{27}$, A. C. C. Lourenço ${ }^{27}$, R. Margutti ${ }^{19}$, J. L. Marshall ${ }^{15}$, T. Matheson ${ }^{28}$, G. E. Medina ${ }^{29}$, B. D. Metzger ${ }^{30}$, R. R. Muñoz ${ }^{29}$, J. Muir ${ }^{31}$, M. Nicholl ${ }^{5}$, E. Quataert ${ }^{32}$, A. Rest ${ }^{33,34}$, M. Sauseda ${ }^{14}$, D. J. Schlegel ${ }^{35}$, L. F. Secco ${ }^{7}$, F. Sobreira ${ }^{36,37}$, A. Stebbins ${ }^{2}$, V. A. Villar ${ }^{6}$, K. Vivas ${ }^{38}$, A. R. Walker ${ }^{38}$, W. Wester ${ }^{2}$,

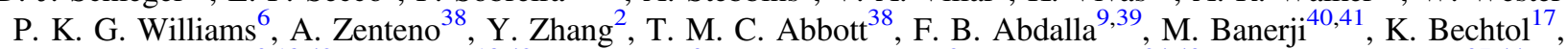
A. Benoit-Lévy ${ }^{9,18,42}$, E. Bertin ${ }^{18,42}$, D. Brooks 9 , E. Buckley-Geer ${ }^{2}$, D. L. Burke ${ }^{24,43}$, A. Carnero Rosell ${ }^{37,44}$, M. Carrasco Kind ${ }^{11,25}$, J. Carretero ${ }^{45}$, F. J. Castander ${ }^{46}$, M. Crocce ${ }^{46}$, C. E. Cunha ${ }^{43}$, C. B. D’Andrea ${ }^{7}$, L. N. da Costa ${ }^{37,44}$, C. Davis ${ }^{43}$, S. Desai ${ }^{47}$, J. P. Dietrich ${ }^{48,49}$, P. Doel ${ }^{9}$, T. F. Eifler ${ }^{50,51}$, E. Fernandez ${ }^{45}$, B. Flaugher ${ }^{2}$, P. Fosalba ${ }^{46}$, E. Gaztanaga ${ }^{46}$, D. W. Gerdes ${ }^{52,53}$, T. Giannantonio ${ }^{40,41,54}$, D. A. Goldstein ${ }^{55,56}$, D. Gruen ${ }^{24,43}$, J. Gschwend ${ }^{37,44}$, G. Gutierrez ${ }^{2}$, K. Honscheid ${ }^{57,58}$, B. Jain ${ }^{7}$, D. J. James ${ }^{59}$, T. Jeltema ${ }^{20}$, M. W. G. Johnson ${ }^{11}$, M. D. Johnson ${ }^{11}$, S. Kent ${ }^{2,4}$, E. Krause ${ }^{43}$, R. Kron ${ }^{2,4}$, K. Kuehn $^{60}$, S. Kuhlmann ${ }^{61}$, O. Lahav ${ }^{9}$, M. Lima ${ }^{37,62}$, M. A. G. Maia ${ }^{37,44}$, M. March ${ }^{7}$, R. G. McMahon ${ }^{40,41}$, F. Menanteau ${ }^{11,25}$, R. Miquel ${ }^{45,63}$, J. J. Mohr ${ }^{48,49,64}$, R. C. Nichol ${ }^{62}$, B. Nord ${ }^{2}$, R. L. C. Ogando ${ }^{37,44}$, D. Petravick ${ }^{11}$, A. A. Plazas ${ }^{51}$, A. K. Romer ${ }^{66}$, A. Roodman ${ }^{24,43}$, E. S. Rykoff ${ }^{24,43}$, E. Sanchez ${ }^{67}$, V. Scarpine ${ }^{2}$, M. Schubnell ${ }^{53}$, I. Sevilla-Noarbe ${ }^{67}$, M. Smith ${ }^{68}$, R. C. Smith ${ }^{38}$, E. Suchyta ${ }^{69}$, M. E. C. Swanson ${ }^{11}$, G. Tarle ${ }^{53}$, D. Thomas ${ }^{65}$, R. C. Thomas ${ }^{56}$, M. A. Troxel ${ }^{57,58}$, V. Vikram ${ }^{61}$, R. H. Wechsler ${ }^{24,43,70}$, and J. Weller ${ }^{48,54,64}$

(The Dark Energy Survey and The Dark Energy Camera GW-EM Collaboration)

\footnotetext{
${ }^{1}$ Department of Physics, Brandeis University, Waltham, MA 02453, USA

${ }^{2}$ Fermi National Accelerator Laboratory, P.O. Box 500, Batavia, IL 60510, USA

${ }^{3}$ Enrico Fermi Institute, Department of Physics, Department of Astronomy and Astrophysics, University of Chicago, Chicago, IL 60637, USA

${ }^{4}$ Kavli Institute for Cosmological Physics, University of Chicago, Chicago, IL 60637, USA

${ }^{5}$ Astrophysical Institute, Department of Physics and Astronomy, 251B Clippinger Lab, Ohio University, Athens, OH 45701, USA

${ }^{6}$ Harvard-Smithsonian Center for Astrophysics, 60 Garden Street, Cambridge, MA 02138, USA

${ }_{8}^{7}$ Department of Physics and Astronomy, University of Pennsylvania, Philadelphia, PA 19104, USA

${ }^{8}$ Department of Astronomy, Indiana University, 727 E. Third Street, Bloomington, IN 47405, USA

${ }^{9}$ Department of Physics \& Astronomy, University College London, Gower Street, London WC1E 6BT, UK

${ }^{10}$ Department of Physics, ETH Zurich, Wolfgang-Pauli-Strasse 16, CH-8093 Zurich, Switzerland

${ }^{11}$ National Center for Supercomputing Applications, 1205 West Clark Street, Urbana, IL 61801, USA

${ }_{13}^{12}$ Department of Physics, University of Surrey, Guildford GU2 7XH, UK

${ }^{13}$ Department of Physics, Syracuse University, Syracuse NY 13224, USA

${ }^{14}$ LSST, 933 North Cherry Avenue, Tucson, AZ 85721, USA

${ }^{15}$ University of Nottingham, School of Physics and Astronomy, Nottingham NG7 2RD, UK

${ }^{16}$ George P. and Cynthia Woods Mitchell Institute for Fundamental Physics and Astronomy, and Department of Physics and Astronomy, Texas A\&M University, College Station, TX 77843, USA

17 The Observatories of the Carnegie Institution for Science, 813 Santa Barbara Street, Pasadena, CA 91101, USA

${ }^{18}$ Institut d'Astrophysique de Paris (UMR7095: CNRS and UPMC), 98 bis Bd Arago, F-75014 Paris, France

Santa Cruz Institute for Particle Physics, Santa Cruz, CA 95064, USA

${ }^{20}$ Center for Interdisciplinary Exploration and Research in Astrophysics (CIERA) and Department of Physics and Astronomy, Northwestern University, Evanston, IL 60208, USA

${ }_{21}^{21}$ Center for Theoretical Astrophysics, Los Alamos National Laboratory, Los Alamos, NM 87544, USA

${ }^{22}$ Instituto de Fisica Teorica UAM/CSIC, Universidad Autonoma de Madrid, E-28049 Madrid, Spain ${ }^{23}$ SLAC National Accelerator Laboratory, Menlo Park, CA 94025, USA

${ }^{24}$ Department of Astronomy, University of Illinois, 1002 W. Green Street, Urbana, IL 61801, USA

${ }^{25}$ Department of Physics and Astronomy \& Astrophysics,The Pennsylvania State University, University Park, PA 16802, USA

${ }_{27}^{26}$ Departments of Physics and Astronomy, and Theoretical Astrophysics Center, University of California, Berkeley, CA 94720-7300, USA

${ }^{27}$ Observatòrio do Valongo, Universidade Federal do Rio de Janeiro, Ladeira do Pedro Antônio 43, Rio de Janeiro, RJ 20080-090, Brazil

${ }^{28}$ National Optical Astronomy Observatory, 950 North Cherry Avenue, Tucson, AZ 85719, USA

${ }^{29}$ Departamento de Astronomonía, Universidad de Chile, Camino del Observatorio 1515, Las Condes, Santiago, Chile

${ }^{30}$ Department of Physics and Columbia Astrophysics Laboratory, Columbia University, New York, NY 10027, USA

${ }^{31}$ Department of Physics, University of Michigan, 450 Church Street, Ann Arbor, MI 48109-1040, USA

${ }^{32}$ Department of Astronomy \& Theoretical Astrophysics Center, University of California, Berkeley, CA 94720-3411, USA

${ }^{33}$ Space Telescope Science Institute, 3700 San Martin Drive, Baltimore, MD 21218, USA

${ }^{34}$ Department of Physics and Astronomy, The Johns Hopkins University, 3400 North Charles Street, Baltimore, MD 21218, USA

35 Physics Division, Lawrence Berkeley National Laboratory, Berkeley, CA 94720-8160, USA

${ }^{36}$ Instituto de Fisica Gleb Wataghin, Universidade Estadual de Campinas, 13083-859, Campinas, SP, Brazil

${ }^{37}$ Laboratório Interinstitucional de e-Astronomia-LIneA, Rua Gal. José Cristino 77, Rio de Janeiro, RJ-20921-400, Brazil

${ }^{38}$ Cerro Tololo Inter-American Observatory, National Optical Astronomy Observatory, Casilla 603, La Serena, Chile

Department of Physics and Electronics, Rhodes University, P.O. Box 94, Grahamstown 6140, South Africa

${ }^{40}$ Institute of Astronomy, University of Cambridge, Madingley Road, Cambridge CB3 OHA, UK
} 


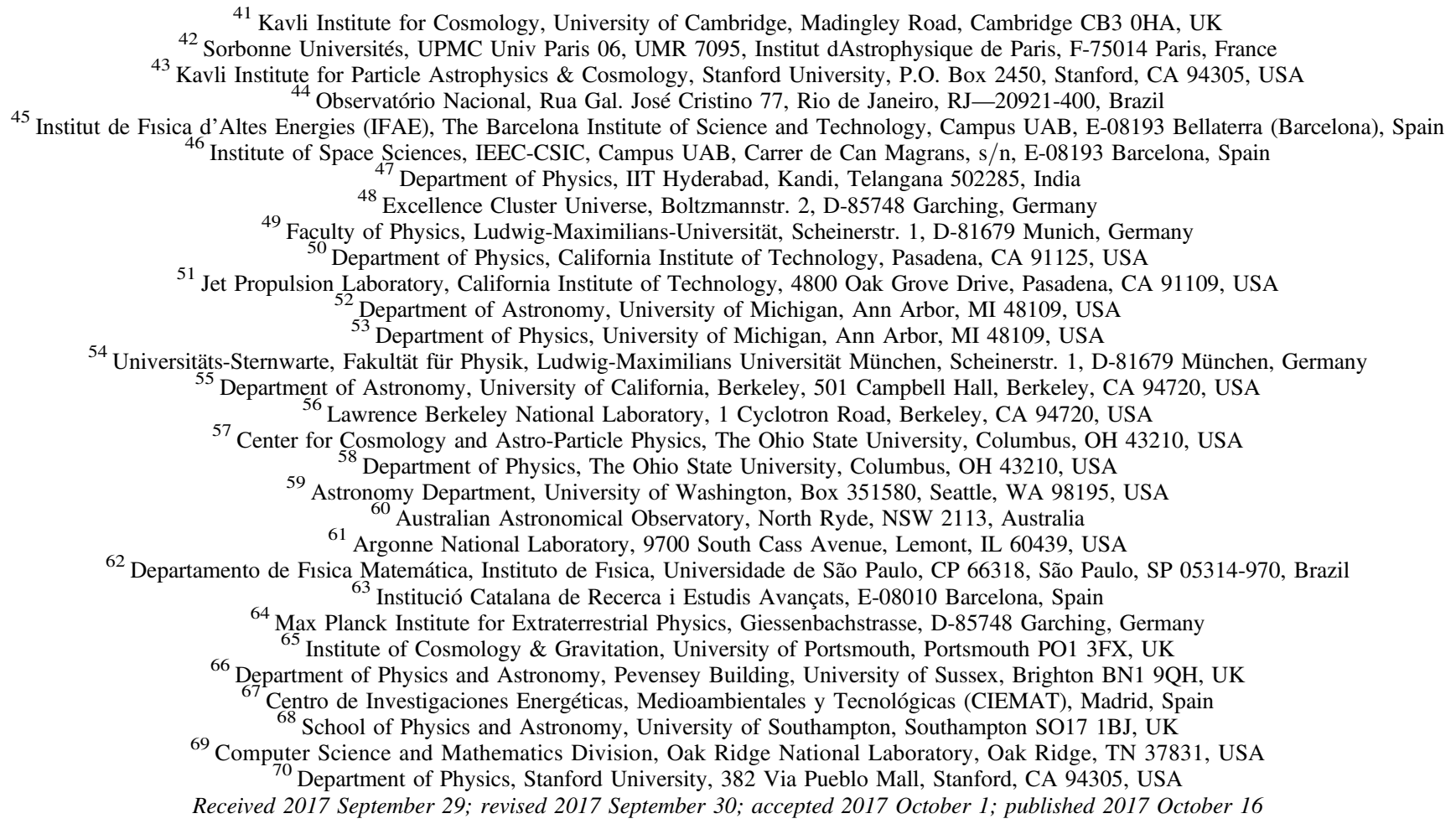

\begin{abstract}
We present the Dark Energy Camera (DECam) discovery of the optical counterpart of the first binary neutron star merger detected through gravitational-wave emission, GW170817. Our observations commenced $10.5 \mathrm{hr}$ post-merger, as soon as the localization region became accessible from Chile. We imaged $70 \mathrm{deg}^{2}$ in the $i$ and $z$ bands, covering 93\% of the initial integrated localization probability, to a depth necessary to identify likely optical counterparts (e.g., a kilonova). At $11.4 \mathrm{hr}$ post-merger we detected a bright optical transient located 10"' 6 from the nucleus of NGC 4993 at redshift $z=0.0098$, consistent (for $H_{0}=70 \mathrm{~km} \mathrm{~s}^{-1} \mathrm{Mpc}^{-1}$ ) with the distance of $40 \pm 8 \mathrm{Mpc}$ reported by the LIGO Scientific Collaboration and the Virgo Collaboration (LVC). At detection the transient had magnitudes of $i=17.3$ and $z=17.4$, and thus an absolute magnitude of $M_{i}=-15.7$, in the luminosity range expected for a kilonova. We identified 1500 potential transient candidates. Applying simple selection criteria aimed at rejecting background events such as supernovae, we find the transient associated with NGC 4993 as the only remaining plausible counterpart, and reject chance coincidence at the $99.5 \%$ confidence level. We therefore conclude that the optical counterpart we have identified near NGC 4993 is associated with GW170817. This discovery ushers in the era of multi-messenger astronomy with gravitational waves and demonstrates the power of DECam to identify the optical counterparts of gravitational-wave sources.
\end{abstract}

Key words: binaries: close - catalogs - gravitational waves - stars: neutron - surveys

Supporting material: machine-readable table

\section{Introduction}

The joint detection of electromagnetic (EM) and gravitational-wave $(\mathrm{GW})$ emission from astrophysical sources is one of the holy grails of present-day astronomy. The primary targets for such joint detections are the mergers of compact object binaries composed of neutron stars (NS) and/or black holes. In such systems, the GW emission provides insight into the bulk motions, masses, binary properties, and potentially the composition of neutron stars. Electromagnetic observations provide critical insights into the astrophysics of the event, such as the progenitor environment, the formation of relativistic and non-relativistic outflows, and in some cases the nature of

\footnotetext{
${ }^{71}$ Hubble and Carnegie-Dunlap Fellow.

72 Hubble Fellow.
}

merger products (e.g., Metzger \& Berger 2012; Rosswog et al. 2013; Baiotti \& Rezzolla 2017). Combining EM and GW observations would lead to deeper scientific insights into some of the most cataclysmic events in the universe. These multimessenger observations also allow for novel measurements, such as standard siren measurements of the Hubble constant (Schutz 1986; Holz \& Hughes 2005; Dalal et al. 2006; Nissanke et al. 2010, 2013), and studies of gamma-ray bursts (GRBs; Berger 2014).

A wide range of EM emission mechanisms for GW sources has been proposed over the years (Metzger \& Berger 2012), including short-duration GRBs (Eichler et al. 1989; Nakar 2007; Berger 2014), on- or off-axis afterglow emission from radio to X-rays (van Eerten \& MacFadyen 2011; Coward et al. 2014; Fong et al. 2015; Lamb \& Kobayashi 2016), optical/near-IR emission due to radioactive decay of $r$-process nuclei 


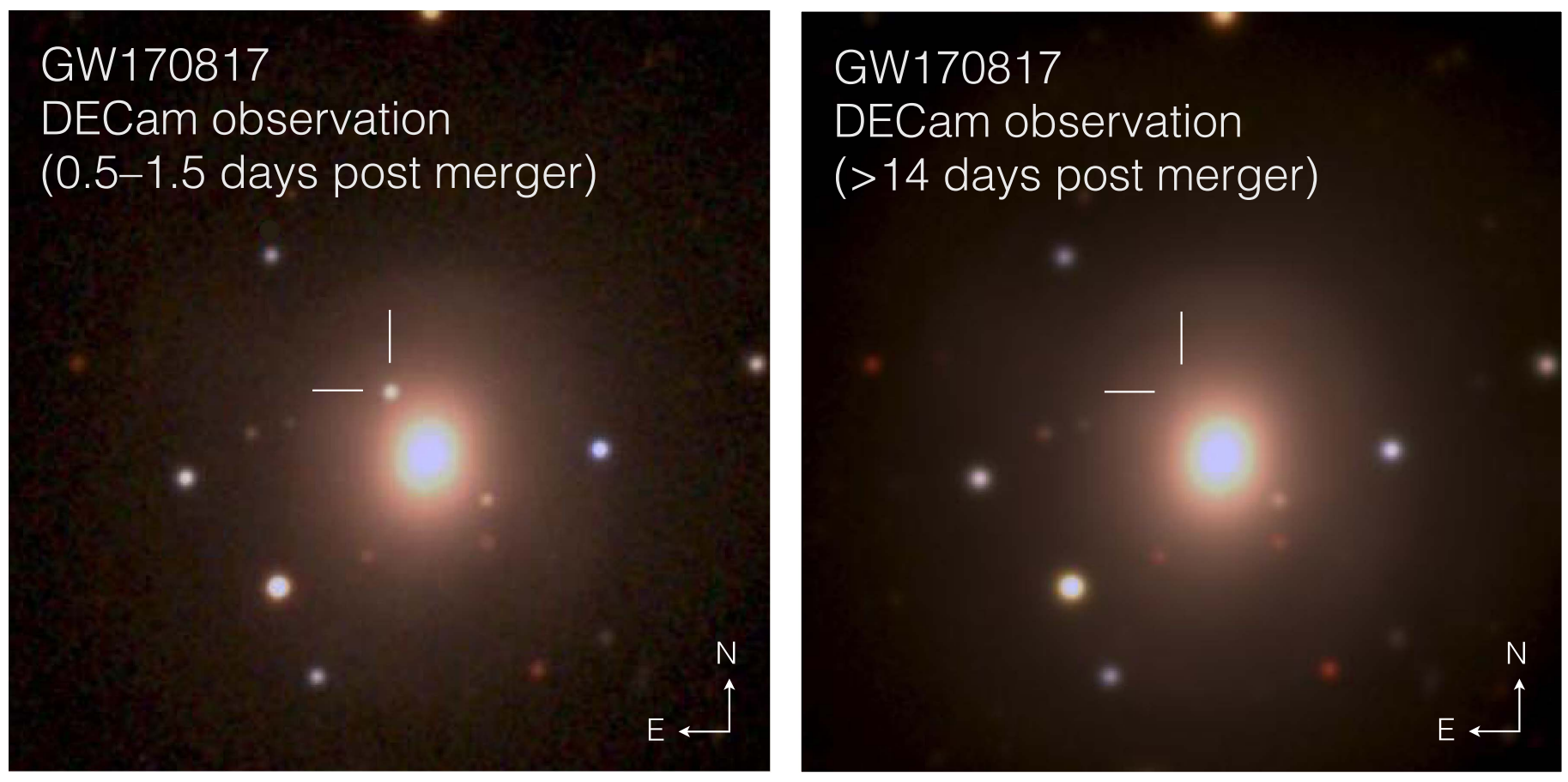

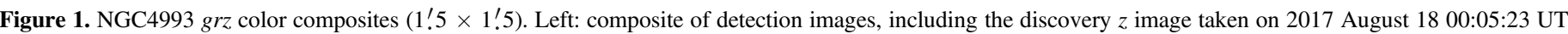

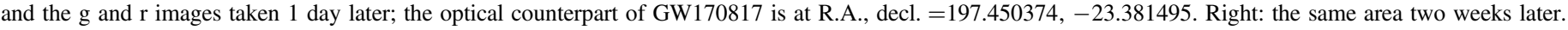

synthesized in the merger ejecta (so-called kilonova; Li \& Paczyński 1998; Rosswog et al. 1999; Metzger et al. 2010; Barnes \& Kasen 2013), and radio emission produced by interaction of the kilonova ejecta with the circumbinary medium (Nakar \& Piran 2011; Metzger \& Berger 2012).

The search for optical counterparts is particularly attractive due to the combination of emission that, unlike GRB emission, is not highly beamed and wide-field optical telescope facilities; a detection can then be followed up at other wavelengths with narrow-field instruments. Over the last two years, we have used the Dark Energy Camera (DECam; Flaugher et al. 2015), a 3 $\mathrm{deg}^{2}$ wide-field imager on the Blanco $4 \mathrm{~m}$ telescope at the Cerro Tololo Inter-American Observatory (CTIO), to follow up GW sources from the advanced Laser Interferometer Gravitational-wave Observatory (aLIGO; Abbott et al. 2009) and Virgo (Acernese et al. 2015) detectors (see, e.g., Abbott et al. 2016b; Dark Energy Survey Collaboration 2016). In particular, we conducted rapid follow-up observations of the black hole binary merger events GW150914 (Abbott et al. 2016c) and GW151226 (Abbott et al. 2016a), using DECam (Annis et al. 2016; Cowperthwaite et al. 2016; Soares-Santos et al. 2016). No optical counterpart was discovered in either case.

On 2017 August 17 at 12:41:06 UT the advanced LIGO/Virgo (ALV) observatories detected a binary neutron star merger, GW170817 (LIGO Scientific Collaboration \& Virgo Collaboration 2017b, 2017d, 2017a). At 23:12:59 UT (10.53 hr after the GW detection) we began to image a $70.4 \mathrm{deg}^{2}$ region that covered $93 \%$ of the localization probability in the map provided by the LVC at the time (LIGO Scientific Collaboration \& Virgo Collaboration 2017c). Immediately following the identification by one of us (R. Chornock), we received a private communication from another DECam team member (R. Foley) indicating that the source was also discovered in an image taken $0.5 \mathrm{hr}$ ahead of ours by the Swope Telescope. We issued a circular to the Gamma-ray Coordination Network (GCN) reporting the discovery at
01:15:01UT (Allam et al. 2017), including a reference to a GCN from the 1M2H Collaboration at 01:05:23 UT (SSS17a; Coulter et al. 2017), and subsequent to our GCN the DLT40 team also announced an independent detection (DLT17ck; Yang et al. 2017 reported at 01:41:13 UT); see LIGO Scientific Collaboration \& Virgo Collaboration et al. (2017a) for an overview of the observations carried out by the community. This transient has received an IAU name of AT2017gfo.

Subsequent to our discovery of the optical transient, we obtained follow-up observations with a wide range of telescopes, spanning radio to $\mathrm{X}$-rays, which are detailed in the associated papers of this series: Cowperthwaite et al. (2017), Nicholl et al. (2017), Chornock et al. (2017), Margutti et al. (2017), Alexander et al. (2017), Blanchard et al. (2017), and Fong et al. (2017).

Here, in the first paper of the series, we present our DECam observations, the discovery of the optical transient, and a search for other potential counterparts across the $70.4 \mathrm{deg}^{2}$ region. We find no other potential optical counterpart within the GW localization region, thus helping to significantly establish the association between the detected optical transient and GW170817. A measurement of the Hubble constant, the first utilizing a gravitational-wave event as a standard siren measurement of distance (Schutz 1986; Dalal et al. 2006), is enabled by this work and is described in LIGO Scientific Collaboration \& Virgo Collaboration et al. (2017b).

\section{DECam Counterpart Search}

The alert for GW170817 was issued 40 minutes after the trigger, on 2017 August 17 at 13:21 UT (Abbott et al. 2017; LIGO Scientific Collaboration \& Virgo Collaboration 2017b), and was promptly received by our automated GCN listener system. Two subsequent GCN circulars indicated that the highsignificance candidate was consistent with a binary neutron star merger at $d \approx 40 \mathrm{Mpc}$ and coincident within $2 \mathrm{~s}$ with a short 


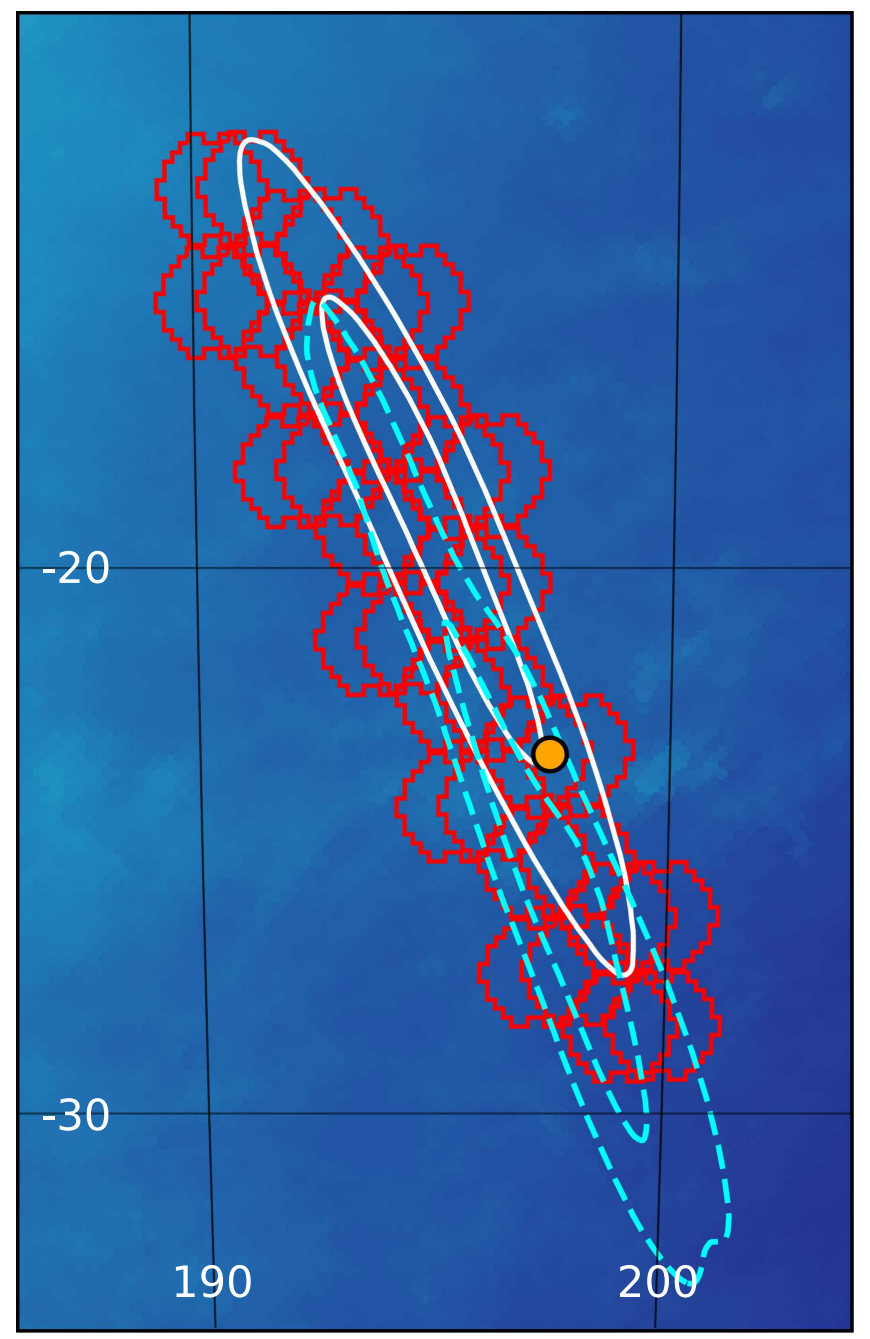

Figure 2. Location of the optical counterpart of GW170817 on the probability maps provided by the LVC (white solid: initial; cyan dashed: revised; inner and outer contours show $50 \%$ and $90 \%$ probability, respectively) and relative to our search area (red: DECam sky footprint).

burst of gamma-rays detected by Fermi-GBM (LIGO Scientific Collaboration \& Virgo Collaboration 2017b, 2017d). Four hours later a sky localization map obtained from the threedetector ALV network was provided (LIGO Scientific Collaboration \& Virgo Collaboration 2017c).

The entire GW localization region was visible from Chile at the beginning of the night, setting within the first $\sim 1.5 \mathrm{hr}$. Our DECam observations commenced at 23:13 UT (10.53 hr postmerger) with $30 \mathrm{~s}$ exposures in the $i$ and $z$ bands. The resulting $5 \sigma$ limiting magnitudes are $i \approx 22.0$ and $z \approx 21.3$ for point sources. The predetermined sequence of observations consisted of 18 pointings (hexes), each with a $3 \mathrm{deg}^{2}$ coverage, with a second offset sequence to mitigate loss of area (e.g., due to gaps between detectors in the focal plane). The resulting areal coverage was 70.4 $\mathrm{deg}^{2}$, corresponding to an integrated probability of $93.4 \%$ of the initial GW skymap. Additional details of the pointing and sequencing determination algorithm are available in Herner et al. (2017). While our sequence of observations was ongoing, a new localization map was released at 23:54 UT (LIGO Scientific Collaboration \& Virgo Collaboration 2017e). While the overall shape of the two maps are similar, the probability peak was shifted significantly. In the revised map, the integrated probability of our observations is $80.7 \%$.

\subsection{Discovery and Observations}

We performed a visual inspection of raw, unprocessed DECam images to find new point sources near relatively bright galaxies in comparison to archival Pan-STARRS1 $3 \pi$ survey images (Chambers et al. 2016). This process resulted in the discovery of a new source near the galaxy NGC 4993 (see Figure 1). The galaxy is located at $z=0.0098$, which is, for a value of $H_{0}$ of $70 \mathrm{~km} \mathrm{~s}^{-1} \mathrm{Mpc}^{-1}$, consistent with the $40 \pm 8$ $\mathrm{Mpc}$ reported by the LVC in their GCN for GW170817. The transient is located at coordinates R.A., decl. $=197.450374$, $-23.381495\left(13^{\mathrm{h}} 09^{\mathrm{m}} 48^{\mathrm{s}} .09-23^{\mathrm{d}} 22^{\mathrm{m}} 53^{\mathrm{s}} .38\right)$ between the $50 \%$ and $90 \%$ contours in both the initial and shifted maps (see Figure 2).

At the time when the galaxy was imaged $(11.40 \mathrm{hr}$ postmerger) the optical transient had magnitudes of $i=17.30 \pm 0.01$ and $z=17.43 \pm 0.01$. We continued to observe the optical counterpart with DECam nightly in the ugrizY filters until it became undetectable (at limiting magnitude $\sim 22.5 \mathrm{mag}$ ) in each band and the source location became inaccessible to the telescope. Our last deep image of the source is on 2017 August 31, 14.5 days post-merger. These follow-up observations are discussed in detail in Cowperthwaite et al. (2017).

We process all images with the DES single-epoch processing (Drlica-Wagner et al. 2017; E. Morganson et al. 2017, in preparation, and references therein) and difference imaging (diffimg) pipelines (Kessler et al. 2015). The diffimg software works by comparing search images and one or more reference images (templates) obtained before or after the search images. We use our own imaging plus publicly available DECam data from the NOAO Science Archive (portal-nvo.noao.edu) as templates, requiring exposures of at least $30 \mathrm{~s}$. At the position of the counterpart, pre-existing templates were available in $g, r$ bands. For $u, i, z, Y$ images we used exposures taken after the source had faded ( $u$ : 2017 August 25; i, z, Y: 2017 August 31).

The photometric results from diffimg are shown in Figure 3 and Table 1. The diffimg pipeline uses the well-tested DES calibration module expCalib. The ugrizY photometry presented in Table 1 has calibration errors relative to DES photometry of $\lesssim 2 \%$. We implemented a galaxy morphological fit and subtraction method, making use of a fast multicomponent fitting software (Imfit; Erwin 2015) followed by point-spread function (PSF) photometry and a Pan-STARRS PS1 calibration to double check the reduction. Results agree within uncertainties and calibration differences. The photometry used in the next paper in this series (Cowperthwaite et al. 2017), measured using a difference image reduction using PanSTARRS PS1 templates, also agrees within uncertainties.

\section{Wide Area Search}

Our primary program is to obtain images over the LIGO probability map to search for counterparts. Within $12 \mathrm{hr}$ of the event we had obtained DECam $i, z$ images across $>80 \%$ of the revised LIGO probability map. We have analyzed this region to determine how many potential counterparts are present.

\subsection{Image Processing}

We employ the DES single-epoch processing and diffimg pipelines to produce a list of transient candidates. The search images are 72 exposures taken on the night of the trigger (corresponding to two tilings of 18 hexes in the $i$ and $z$ bands). Because most of the exposures $(\sim 60 \%)$ did not have pre- 


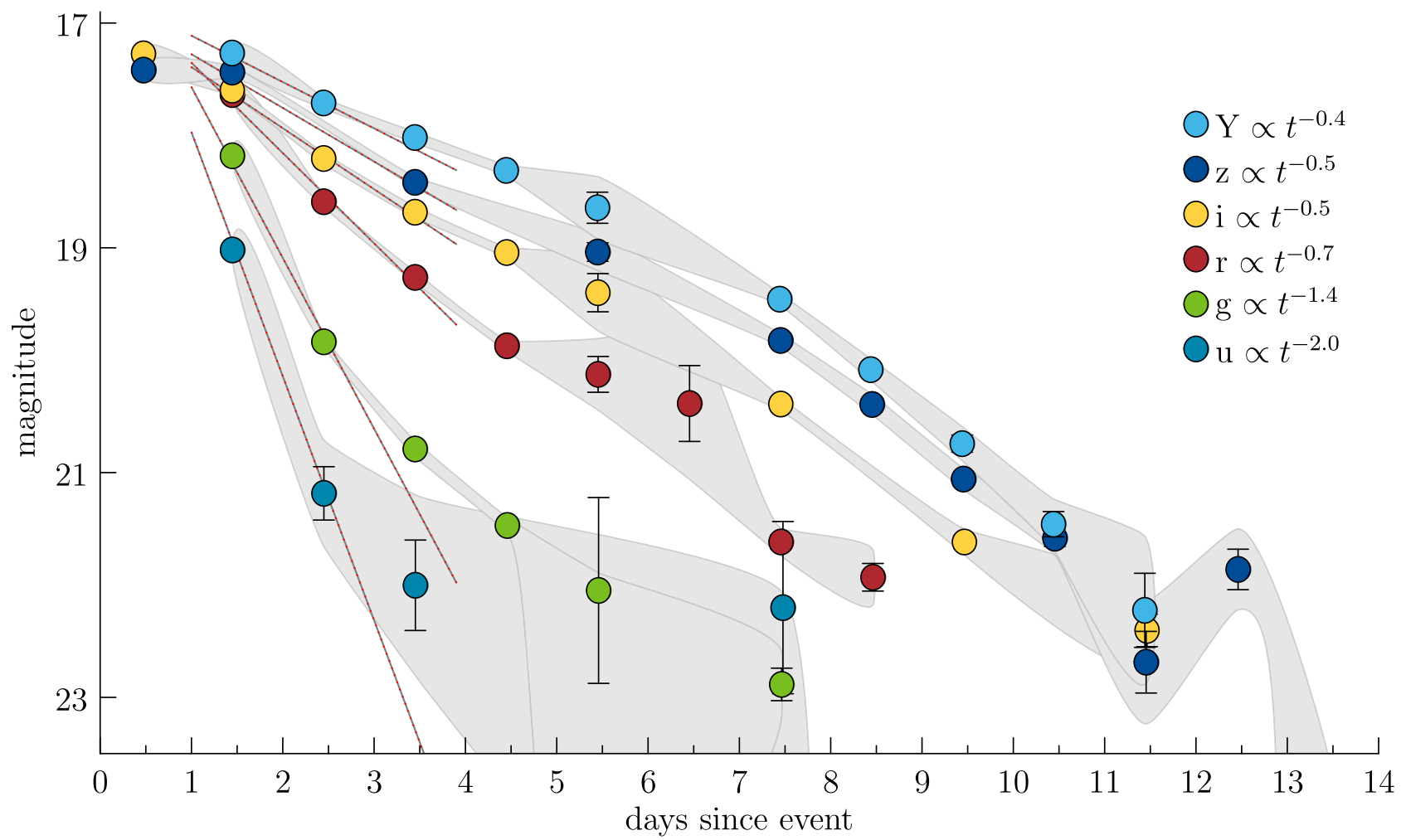

Figure 3. Observed light curve for the optical counterpart of GW170817 measured in six filters. The data points and $1 \sigma$ error bars were measured using diffimg. The gray bands represent the $95 \%$ confidence interval about each measurement, or $2 \sigma$ above sky if the measured flux is less than $2 \sigma_{\text {sky }}$. Data taken on days 5.5 , 6.5 , and 11.5 were obtained in poor weather conditions, hence the broader uncertainty bands. The photometry has been checked against a difference imaging reduction using Pan-STARRS PS1 templates (Cowperthwaite et al. 2017) and a galaxy fit and subtract PSF source magnitude reduction; these measurements are consistent with our results. The dashed lines show the initial decay of the source: $m \propto t^{-\alpha}$, where $\alpha=(2.0,1.4,0.7,0.5,0.5,0.4)$ in u, $\mathrm{g}, \mathrm{r}, \mathrm{i}, \mathrm{z}, \mathrm{Y}$ bands, respectively. The decay in the first few days is consistent with a peak luminosity near $t=1$ day. The table containing light curve information is available as a machine-readable file (Table 1 ).

Table 1

Light Curve Constructed from u, g, r, i, z, Y Observations

\begin{tabular}{lccc}
\hline \hline MJD & Band & Mag $^{\mathrm{a}}$ & $\sigma_{m}$ \\
\hline 57983.00306 & $\mathrm{i}$ & 17.27 & 0.005 \\
57983.00374 & $\mathrm{Z}$ & 17.42 & 0.007 \\
57983.97395 & $\mathrm{Y}$ & 17.27 & 0.008 \\
\hline
\end{tabular}

Note. Columns are observation time, band, magnitude, and its errors Magnitudes are galactic extinction corrected AB PSF magnitudes.

${ }^{\mathrm{a}} E(B-V)_{\mathrm{SFD} 98}=0.123$, and $R_{V}=3.963,3.186,2.140,1.569,1.196,1.048$ for $\mathrm{u}, \mathrm{g}, \mathrm{r}, \mathrm{i}, \mathrm{z}, \mathrm{Y}$ bands, respectively.

(This table is available in its entirety in machine-readable form.)

Table 2

Number of Candidates at Each Selection Stage, Sorted by $i$-band Magnitude

\begin{tabular}{lrrcc}
\hline \hline $\operatorname{mag}(i)$ & Raw & Cut 1 & Cut 2 & Cut 3 \\
\hline $15.5-16.5$ & 4 & 0 & 0 & 0 \\
$16.5-17.5$ & 11 & 7 & 3 & 1 \\
$17.5-18.5$ & 26 & 15 & 7 & 0 \\
$18.5-19.5$ & 296 & 63 & 27 & 0 \\
$19.5-20.5$ & 1163 & 167 & 44 & 0 \\
\hline Total & 1500 & 252 & 81 & 1 \\
\hline
\end{tabular}

existing DECam imaging in those bands, we took images on the nights of August 31 through September 2 to serve as templates. We expect any kilonova-like transients to have faded below detection thresholds by that time ( $\sim 2$ weeks from the trigger).

The total area encompassed by the 72 exposures is $70.4 \mathrm{deg}^{2}$. The camera fill factor is $80 \%$, which results in $4 \%$ area loss when we consider the two overlapping tilings. Weather on the nights of template observations was partially clouded and caused a 3\% loss. Processing failures accounted for less than $1 \%$ loss. The final area included in this search for other potential counterparts is $64.6 \mathrm{deg}^{2}$.

This process results in 1500 transient candidates with magnitudes between 15.5 and 20.5. A candidate is defined as a detection meeting diffimg quality requirements (see Table 3 of Kessler et al. 2015) on at least two search exposures. The magnitude cutoff of this analysis is limited by the depth of the template images: $i=21.2$ and $z=20.5$, to be compared with the depth of the search images: $i=22.0$ and $z=21.3$.

\subsection{Candidate Selection}

We apply several selection criteria to candidates identified in diffimg aiming to reject moving objects, background artifacts, and long-lived transients:

1. Criterion 1: the candidate must have at least one detection in $i$ and one detection in the $z$ band.

2. Criterion 2: the candidate must pass our automated scanning program (Goldstein et al. 2015) with a machinelearning score $\geqslant 0.7$ in all detections. This criterion rejects non-astrophysical artifacts. The efficiency of this criterion 
as measured from point sources injected into our images is $\approx 90 \%-100 \%$ for both $i$ and $z \approx 17-22$ mag.

3. Criterion 3: the candidate must have faded significantly between the search (first) and template (last) observations. Specifically, we require the change in the candidate flux to be greater, at $3 \sigma$ level, than the flux in a circular aperture of radius $=5$ pixels on the template image at the location of the candidate. This criterion eliminates slowevolving transients (e.g., supernovae).

Table 2 lists the number of events passing each selection stage in various bins of magnitude. After all criteria are applied, one optical counterpart candidate remains: the source discovered by visual inspection. Slow moving solar system objects, which could potentially have met the selection criteria above, are very rare in the magnitude range of this search. The flares of $\mathrm{M}$ dwarf flare stars have $T \approx 10,000 \mathrm{~K}$ and therefore are very blue; they are rejected by our selection criterion 3 in the $\mathrm{z}$ band.

\section{Uniqueness of the Candidate}

This analysis shows that the source we discovered is the only one plausibly associated with the GW event within the region searched. To estimate its significance we compute the chance probability of a transient to occur within the volume and timescale of interest. Because SNe are by far the most likely transient contaminant, we use their rate and timescale to make a conservative estimate. We use a combined rate of $1 \times 10^{-4}$ $\mathrm{Mpc}^{-3} \mathrm{yr}^{-1}$, for core-collapse (Strolger et al. 2015) and Type Ia (Dilday et al. 2008) $\mathrm{SNe}$ at $z \lesssim 0.1$. The characteristic timescale of SNe is $\tau \sim 1$ month. The volume $(V)$ we observed is estimated as a shell at $z \sim 0.01(\sim 40 \mathrm{Mpc})$ spanning a 64 $\mathrm{deg}^{2}$ area and $16 \mathrm{Mpc}$ width corresponding to the effective search area and the distance uncertainty, respectively: $V=558$ $\mathrm{Mpc}^{3}$. Under these assumptions, we find that the probability of a chance coincidence is $\sim 0.5 \%$, and we conclude that our optical transient is associated with GW170817.

\section{Conclusion}

We report the DECam discovery of the optical counterpart to the BNS merger GW170817, an object with $i=17.30$ mag and $z=17.43 \mathrm{mag}$ at $11.40 \mathrm{hr}$ post-merger. The source was discovered through visual inspection of nearby galaxies in our raw data stream. Our analysis identifies this source as the only credible optical counterpart within a large fraction of the GW170817 skymap. The observed peak absolute magnitude of $M_{i}=-15.7$ is about 1000 times brighter than a nova, which is typically close to Eddington luminosity $\left(M_{V}=-9\right)$. Thus, we have indeed discovered a kilonova as the name defines it and was predicted in Metzger et al. (2010).

At $M_{i}=-15.7$, the optical transient is bright enough for us to detect it out to $425 \mathrm{Mpc}$. Its properties, 1.5 days after the event include: $(i-z)=0.2$, and a magnitude decline versus time in $i, z, Y \propto t^{-\frac{1}{2}}$, and faster decline in the $g$ band $\left(\propto t^{-\frac{3}{2}}\right)$. Future searches for counterparts of $\mathrm{GW}$ events may be improved by this information.

This detection has opened a new era of multi-probe, multimessenger astronomical observations of the universe that will bring new measurements of cosmological parameters, starting with the present rate of expansion (LIGO Scientific Collaboration \& Virgo Collaboration et al. 2017b), and possibly helping determine the matter/energy content and evolution of the universe.

This is the first detection of an optical counterpart of a gravitational-wave source. It will not be the last. As the LIGO and Virgo Collaborations proceed to their next observing runs and upgrades, DECam will continue to play an important, almost unique, role in the identification of gravitational-wave sources in the Southern Hemisphere.

Funding for the DES Projects has been provided by the DOE and NSF (USA), MEC/MICINN/MINECO (Spain), STFC (UK), HEFCE (UK). NCSA (UIUC), KICP (U. Chicago), CCAPP (Ohio State), MIFPA (Texas A\&M), CNPQ, FAPERJ, FINEP (Brazil), DFG (Germany), and the Collaborating Institutions in the Dark Energy Survey.

The Collaborating Institutions are Argonne Lab, UC Santa Cruz, University of Cambridge, CIEMAT-Madrid, University of Chicago, University College London, DES-Brazil Consortium, University of Edinburgh, ETH Zürich, Fermilab, University of Illinois, ICE (IEEC-CSIC), IFAE Barcelona, Lawrence Berkeley Lab, LMU München and the associated Excellence Cluster Universe, University of Michigan, NOAO, University of Nottingham, Ohio State University, University of Pennsylvania, University of Portsmouth, SLAC National Lab, Stanford University, University of Sussex, Texas A\&M University, and the OzDES Membership Consortium.

Based in part on observations at Cerro Tololo InterAmerican Observatory, National Optical Astronomy Observatory, which is operated by the Association of Universities for Research in Astronomy (AURA) under a cooperative agreement with the National Science Foundation.

The DES Data Management System is supported by the NSF under grant numbers AST-1138766 and AST-1536171. The DES participants from Spanish institutions are partially supported by MINECO under grants AYA2015-71825, ESP2015-88861, FPA2015-68048, and Centro de Excelencia SEV-2012-0234, SEV-2016-0597 and MDM-2015-0509. Research leading to these results has received funding from the ERC under the EU's 7th Framework Programme including grants ERC 240672, 291329 and 306478. We acknowledge support from the Australian Research Council Centre of Excellence for All-sky Astrophysics (CAASTRO), through project number CE110001020.

This Letter has been authored by Fermi Research Alliance, LLC under Contract No. DE-AC02-07CH11359 with the U.S. Department of Energy, Office of Science, Office of High Energy Physics. The United States Government retains and the publisher, by accepting the article for publication, acknowledges that the United States Government retains a nonexclusive, paid-up, irrevocable, world-wide license to publish or reproduce the published form of this manuscript, or allow others to do so, for United States Government purposes.

H.Y.C., Z.D., B.F., M.F., and D.E.H. were partially supported by NSF CAREER grant PHY-1151836 and NSF grant PHYS-1708081. They were also supported by the Kavli Institute for Cosmological Physics at the University of Chicago through NSF grant PHY-1125897 and an endowment from the Kavli Foundation.

The Berger Time-Domain Group at Harvard is supported in part by the NSF through grants AST-1411763 and AST1714498, and by NASA through grants NNX15AE50G and NNX16AC22G. 
We thank the University of Copenhagen, DARK Cosmology Centre, and the Niels Bohr International Academy for hosting R.J.F., Z.D., and B.F. during the discovery of GW170817/ SSS17a, where they were participating in the Kavli Summer Program in Astrophysics, "Astrophysics with gravitational wave detections." This program was supported by the Kavli Foundation, Danish National Research Foundation, the Niels Bohr International Academy, and the DARK Cosmology Centre.

The UCSC group is supported in part by NSF grant AST1518052, the Gordon \& Betty Moore Foundation, the HeisingSimons Foundation, generous donations from many individuals through a UCSC Giving Day grant, and from fellowships from the Alfred P. Sloan Foundation and the David and Lucile Packard Foundation to R.J.F.

We thank the CTIO director, Stephen Heathcote, for being supportive of our target of opportunity program using DECam. We also thank the CTIO staff for providing a great experience to observers on-site and remotely.

\section{References}

Abbott, B. P., Abbott, R., Abbott, T. D., et al. 2016a, PhRvL, 116, 241103 Abbott, B. P., Abbott, R., Abbott, T. D., et al. 2016b, ApJL, 826, L13 Abbott, B. P., Abbott, R., Abbott, T. D., et al. 2016c, PhRvL, 116, 061102 Abbott, B. P., Abbott, R., Abbott, T. D., et al. 2017, PhRvL, https://doi.org/ 10.1103/PhysRevLett.119.161101

Abbott, B. P., Abbott, R., Adhikari, R., et al. 2009, RPPh, 72, 076901

Acernese, F., Agathos, M., Agatsuma, K., et al. 2015, CQGra, 32, 024001

Alexander, K., Berger, E., Fong, W., et al. 2017, ApJL, https://doi.org/10.3847/ 2041-8213/aa905d

Allam, S., Annis, J., Berger, E., et al. 2017, GCN, 21530

Annis, J., Soares-Santos, M., Berger, E., et al. 2016, ApJL, 823, L34

Baiotti, L., \& Rezzolla, L. 2017, RPPh, 80, 096901

Barnes, J., \& Kasen, D. 2013, ApJ, 775, 18

Berger, E. 2014, ARA\&A, 52, 43

Blanchard, P. K., Berger, E., Fong, W., et al. 2017, ApJL, https://doi.org/ 10.3847/2041-8213/aa9055

Chambers, K. C., Magnier, E. A., Metcalfe, N., et al. 2016, arXiv:1612.05560

Chornock, R., Berger, E., Kasen, D., et al. 2017, ApJL, https://doi.org/ 10.3847/2041-8213/aa905c

Coulter, D. A., Kilpatrick, C. D., Siebert, M. R., et al. 2017, GCN, 21529

Coward, D. M., Branchesi, M., Howell, E. J., Lasky, P. D., \& Böer, M. 2014, MNRAS, 445, 3575

Cowperthwaite, P. S., Berger, E., Soares-Santos, M., et al. 2016, ApJL, 826, L29
Cowperthwaite, P., Berger, E., Villar, V. E., et al. 2017, ApJL, https://doi. org / 10.3847/2041-8213/aa8fc7

Dalal, N., Holz, D. E., Hughes, S. A., \& Jain, B. 2006, PhRvD, 74, 063006

Dark Energy Survey Collaboration 2016, MNRAS, 460, 1270

Dilday, B., Kessler, R., Frieman, J. A., et al. 2008, ApJ, 682, 262

Drlica-Wagner, A., Sevilla-Noarbe, I., Rykoff, E. S., et al. 2017, arXiv:1708. 01531

Eichler, D., Livio, M., Piran, T., \& Schramm, D. N. 1989, Natur, 340, 126

Erwin, P. 2015, ApJ, 799, 226

Flaugher, B., Diehl, H. T., Honscheid, K., et al. 2015, AJ, 150, 150

Fong, W., Berger, E., Margutti, R., \& Zauderer, B. A. 2015, ApJ, 815, 102

Fong, W., Berger, E., Blanchard, P. K., et al. 2017, ApJL, https://doi.org/ 10.3847/2041-8213/aa9018

Goldstein, D. A., D’Andrea, C. B., Fischer, J. A., et al. 2015, AJ, 150, 82

Herner, K., et al. 2017, in XXII Int. Conf. on Computing in High Energy and Nuclear Physics (CHEP 2016) (San Francisco, CA)

Holz, D. E., \& Hughes, S. A. 2005, ApJ, 629, 15

Kessler, R., Marriner, J., Childress, M., et al. 2015, AJ, 150, 172

Lamb, G. P., \& Kobayashi, S. 2016, ApJ, 829, 112

Li, L.-X., \& Paczyński, B. 1998, ApJL, 507, L59

LIGO Scientific Collaboration \& Virgo Collaboration et al. 2017a, ApJL, https://doi.org/10.3847/2041-8213/aa91c9

LIGO Scientific Collaboration \& Virgo Collaboration et al. 2017b, Natur, https://doi.org/10.1038/nature24471

LIGO Scientific Collaboration \& Virgo Collaboration 2017a, ApJL, https:// doi.org/10.3847/2041-8213/aa920c

LIGO Scientific Collaboration \& Virgo Collaboration 2017b, GCN, 21505

LIGO Scientific Collaboration \& Virgo Collaboration 2017c, GCN, 21513

LIGO Scientific Collaboration \& Virgo Collaboration 2017d, GCN, 21509

LIGO Scientific Collaboration \& Virgo Collaboration 2017e, GCN, 21527

Margutti, R., Berger, E., Fong, W., et al. 2017, ApJL, https://doi.org/ $10.3847 / 2041-8213 /$ aa9057

Metzger, B. D., \& Berger, E. 2012, ApJ, 746, 48

Metzger, B. D., Martínez-Pinedo, G., Darbha, S., et al. 2010, MNRAS, 406, 2650

Nakar, E. 2007, PhR, 442, 166

Nakar, E., \& Piran, T. 2011, Natur, 478, 82

Nicholl, M., Berger, E., Kasen, D., et al. 2017, ApJL, https://doi.org/ 10.3847/2041-8213/aa9029

Nissanke, S., Holz, D. E., Dalal, N., et al. 2013, arXiv:1307.2638

Nissanke, S., Holz, D. E., Hughes, S. A., Dalal, N., \& Sievers, J. L. 2010, ApJ, 725,496

Rosswog, S., Liebendörfer, M., Thielemann, F.-K., et al. 1999, A\&A, 341, 499

Rosswog, S., Piran, T., \& Nakar, E. 2013, MNRAS, 430, 2585

Schutz, B. F. 1986, Natur, 323, 310

Soares-Santos, M., Kessler, R., Berger, E., et al. 2016, ApJL, 823, L33

Strolger, L.-G., Dahlen, T., Rodney, S. A., et al. 2015, ApJ, 813, 93

van Eerten, H. J., \& MacFadyen, A. I. 2011, ApJL, 733, L37

Yang, S., Valenti, S., Sand, D., et al. 2017, GCN, 21531 